\title{
Mast Cell Infiltration Is Involved in Renal Interstitial Fibrosis in a Rat Model of Protein-Overload Nephropathy
}

\author{
Ying $\mathrm{Li}^{1}$ Letian Zhou' ${ }^{1}$ Fuyou Liu Youming Peng Jun Li Lin Sun \\ Shaobin Duan Guanghui Ling Xin Chen Wenling Jiang Yuncheng Xia \\ Division of Nephrology, Second Xiangya Hospital, Research Institute of Nephrology, Central South University, \\ Key Laboratory of Nephrology and Blood Purification in Hunan, Changsha, PR China
}

\section{Key Words}

Protein-overload nephropathy - Mast cell · Stem cell factor ·

Transforming growth factor- $\beta_{1} \cdot$ Renal interstitial fibrosis

\begin{abstract}
Aims: The present study investigated the relationship between mast cells (MCs) and the protein expression of stem cell factor (SCF) and transforming growth factor- $\beta_{1}$ (TGF- $\left.\beta_{1}\right)$ in the regions of renal interstitial fibrosis with protein-overload nephropathy, in order to provide a good animal model to study the mechanism of renal fibrosis induced by proteinuria. Methods: 60 male Sprague-Dawley rats were divided into a bovine serum albumin (BSA) group and a control group. The intensity of MCs infiltration was examined by toluidine blue and chymase and tryptase staining. The protein expression of SCF and TGF- $\beta_{1}$ was respectively examined by immunohistochemical and immunofluorescence staining. Results: Severe proteinuria was induced in rats of the BSA group. Expression of SCF and TGF- $\beta_{1}$ was detected in the tubular and the interstitial cells. The number of MCs positively correlated with the severity of interstitial lesions and the expression of SCF and TGF- $\beta_{1}$. Conclusion: Our results demonstrated that in protein-overload nephropathy, MCs infiltrat-
\end{abstract}

ed into the kidney, and the expression of SCF and TGF- $\beta_{1}$ gradually increased. They might play important roles in the development of renal interstitial fibrosis, but the underlying mechanism needs to be further studied.

Copyright $\odot 2010$ S. Karger AG, Basel

\section{Introduction}

Tubulointerstitial fibrosis is a final common pathway to end-stage renal disease, however, its mechanism is still poorly understood. The extent of tubulointerstitial fibrosis is believed to more possibly predict the decline in renal function and poor prognosis rather than the degree of glomerular damage $[1,2]$. Recent studies suggested that proteins filtered through the glomerular capillary, previously considered as markers of the severity of renal lesions, have intrinsic toxicity on the proximal tubular cells and can directly trigger tubulointerstitial fibrosis [3]. Nevertheless, tubulointerstitial fibrosis is usually preceded by tubulointerstitial infiltration of mononuclear in-

Y.L. and L.Z. contributed equally to this work.

\section{KARGER}

Fax +41613061234 E-Mail karger@karger.ch www.karger.com
(C) 2010 S. Karger AG, Basel

$1420-4096 / 10 / 0333-0240 \$ 26.00 / 0$

Accessible online at:

www.karger.com/kbr
Liu Fuyou, MD or Li Ying, MD

Division of Nephrology, Second Xiangya Hospital

No.139 Renming Road, Changsha, Hunan 410011 (PR China)

Tel./Fax +86731 85881410

E-Mail xyesk@yahoo.com.cn or liyingdoctor@yahoo.com.cn 
flammatory cells. Proteinuria provokes a multitude of inflammatory and fibrogenic mediators, which contribute to the influx of mononuclear cells. Inflammatory cells in return can secrete cytokines and exacerbate tubulointerstitial damage $[4,5]$.

While contemporary research recently focused on the functions of renal tissue macrophages and Tlymphocytes for this pathological process, only little has been said as to the contribution of the mast cells (MCs). MCs, derived from CD34-positive multipotent bone marrow progenitor cells, circulate in blood and migrate to areas of inflammation. The cytoplasmatic granules in MCs contain an array of mediators that influence resident and infiltrating cells in a paracrine manner. These mediators include MC-specific proteases, such as tryptase and chymase, as well as a number of profibrotic acting cytokines. MCs are best known for their traditional role in type I allergies with immunoglobulin E-mediated diseases such as bronchial asthma and anaphylactic reactions $[6,7]$. It has been recently reported that $\mathrm{MC}$ infiltration contributes to the process of tubulointerstitial fibrosis in various renal diseases $[8,9]$. However, the actual role of MCs in the process of renal fibrosis associated with proteinuria has not been fully elucidated. A number of factors responsible for this directional migration and tissue maturation of MCs have been identified, such as stem cell factor (SCF) [10] and transforming growth factor- $\beta_{1}$ $\left(\right.$ TGF- $\left.\beta_{1}\right)$ [11]. On the other hand, SCF and TGF- $\beta_{1}$ can further promote the progression of renal fibrosis.

The present study was designed to investigate the relationship between the infiltration of MCs in kidney with renal interstitial fibrosis and the protein expression of SCF and TGF- $\beta_{1}$ in an animal model with protein-overload nephropathy. This will provide a theoretical foundation and a better animal model to further study the mechanism and therapy.

\section{Methods}

\section{Experimental Protein-Overload Nephropathy Model}

A total of 60 male Sprague-Dawley rats weighing 150-200 g with negative tests of urinary protein and glucose were included in this study. All 60 rats had their right-sided kidney removed. One week following right-sided nephrectomy, rats were randomly divided into a bovine serum albumin (BSA) group and a control group, with 30 rats in each. In the BSA group, protein-overload nephropathy was induced as described previously [12], and the rats received intraperitoneal injections of BSA. The BSA (Proliant Co., USA) was dissolved in saline and the final concentration of BSA was $0.33 \mathrm{~g} / \mathrm{ml}$. The dosage of BSA was $1 \mathrm{ml}$ on the first day and increased gradually to $3 \mathrm{ml} 5$ days later. Then, only rats with 24-hour proteinuria $>8 \mathrm{mg}$ were included in this study and at the end of the study, they were given $5 \mathrm{~g} \mathrm{BSA} / \mathrm{kg}$ body weight. The rats in the control group were intraperitoneally injected with an equal volume of saline. All rats were housed in a temperature-controlled room at $22^{\circ} \mathrm{C}$ with ad libitum access to commercial standard rat food and water during the entire study. A total of 10 rats from the BSA group and control group were sacrificed at 3, 7 and 11 weeks after injection. The animal care protocols in this study were approved in advance by an appropriate animal care and use committee.

Urine and Blood Measurements

24-Hour urine samples were collected for urinary protein quantitation using the Bio-Rad method after 3, 7 and 11 weeks of treatment. Body weight was measured weekly. Blood samples were obtained from the abdominal aorta at sacrifice and were used for measuring serum creatinine and albumin by automatic biochemistry analyzer.

\section{Renal Histology}

A portion of the left kidney was removed from the animal and fixed with $4 \%$ paraformaldehyde, then embedded in paraffin for light-microscopic evaluation. The $3-\mu \mathrm{m}$ sections were cut and stained with hematoxylin and eosin, periodic acid-Schiff reagent, Masson's trichrome, and periodic acid-silver methenamine. Interstitial fibrosis was assessed in sections of kidneys stained with periodic acid-silver methenamine. Sections were coded by an investigator, then digitally photographed and analyzed by another blinded investigator. Photographs of each section were taken using a stereomicroscope fitted with a digital camera (CX41 light microscopy; Olympus, Japan). Tubulointerstitial injuries, seen as tubular dilation and/or atrophy, interstitial fibrosis and inflammatory cell infiltration were graded by the following semiquantitative score: grade 0: no changes; grade 1: focal changes involving $25 \%$ of the sample; grade 2: changes affecting $25-50 \%$; grade 3 : changes involving $50-75 \%$, and grade 4 : lesions affecting $75 \%$ or more. These examinations evaluated the areas overlying the tubular basement membrane and interstitial space, but avoided the glomeruli and large vessels. Twenty cortical tubulointerstitial fields were randomly selected and assessed by an observer who was blinded to the experimental groups, and the mean values were calculated for each specimen.

Histochemical Staining

To identify MCs in the kidneys, toluidine blue (TB) staining was performed by immersion of sections at $\mathrm{pH} 0.5(0.5 \% \mathrm{~TB}$ in $0.5 \mathrm{~N} \mathrm{HCl}$ ) (Sigma Chemical Co., St. Louis, Mo., USA) for 5 days at room temperature.

\section{Immunohistochemical Staining}

Paraffin-embedded tissue sections were dewaxed, hydrated, treated with $5 \mathrm{mmol} / \mathrm{l}$ levamisole for blocking endogenous alkaline phosphatase and incubated with blocking serum for $30 \mathrm{~min}$ at room temperature to reduce nonspecific background staining. Sections were rehydrated in phosphate-buffered saline/0.1\% BSA for 15 min before addition of the appropriate blocking serum for an additional $15 \mathrm{~min}$. Sections were incubated with polyclonal rabbit anti-MC chymase 1:1,000 (Chemicon, Temecula, Calif., USA), rabbit anti-TGF- $\beta_{1} 15 \mu \mathrm{g} / \mathrm{ml}$ (Santa Cruz Biotechnology, Santa Cruz, Calif., USA), and rabbit anti-SCF 1:150 (Santa Cruz 
Table 1. Clinical parameters and morphology changes in BSA-injected rats and saline-injected controls (mean \pm SD)

\begin{tabular}{|c|c|c|c|c|c|c|}
\hline & \multicolumn{2}{|l|}{3 weeks } & \multicolumn{2}{|l|}{7 weeks } & \multicolumn{2}{|l|}{11 weeks } \\
\hline & control & BSA group & control & BSA group & control & BSA group \\
\hline Number & 10 & 10 & 10 & 8 & 10 & 8 \\
\hline Body weight, $g$ & $253.7 \pm 20.8$ & $243.8 \pm 19.3$ & $311.1 \pm 30.4$ & $306.3 \pm 31.4$ & $389.3 \pm 32.8$ & $374.9 \pm 26.9$ \\
\hline Urinary protein, $\mathrm{mg} / 24 \mathrm{~h}$ & $4.0 \pm 1.8$ & $79.0 \pm 28.6^{\mathrm{a}}$ & $4.7 \pm 1.8$ & $199.1 \pm 98.4^{\mathrm{a}, \mathrm{c}}$ & $5.5 \pm 2.4$ & $133.7 \pm 67.8^{a}$ \\
\hline Serum albumin, $g / 1$ & $30.2 \pm 2.7$ & $20.8 \pm 2.2^{\mathrm{a}}$ & $28.9 \pm 2.7$ & $18.4 \pm 2.4^{\mathrm{a}, \mathrm{b}}$ & $27.1 \pm 2.5$ & $15.9 \pm 1.7^{\mathrm{a}, \mathrm{d}}$ \\
\hline Serum creatinine, $\mu \mathrm{mol} / \mathrm{l}$ & $21.8 \pm 3.1$ & $30.5 \pm 11.1$ & $24.1 \pm 3.3$ & $34.5 \pm 7.9$ & $28.3 \pm 5.9$ & $41.8 \pm 11.1$ \\
\hline Tubulointerstitial injury & $0.56 \pm 0.18$ & $1.75 \pm 0.23^{\mathrm{a}}$ & $0.75 \pm 0.21$ & $2.04 \pm 0.44^{\mathrm{a}, \mathrm{c}}$ & $0.78 \pm 0.24$ & $2.89 \pm 0.33^{\mathrm{a}, \mathrm{c}, \mathrm{d}}$ \\
\hline
\end{tabular}

${ }^{a} \mathrm{p}<0.01$ vs. saline-injected controls; ${ }^{\mathrm{b}} \mathrm{p}<0.05 ;{ }^{\mathrm{c}} \mathrm{p}<0.01$ vs. BSA-injected rats at the $3 \mathrm{rd}$ week; ${ }^{\mathrm{d}} \mathrm{p}<0.05$ vs. BSA-injected rats at the 7 th week.

Biotechnology) overnight at $4^{\circ} \mathrm{C}$. After rinsing, the sections were incubated with biotinylated goat anti-rabbit IgG (Abcam, UK) and processed using an alkaline phosphatase-streptavidin-biotin immunoperoxidase method (Beijing Zhongshan Biotechnology Co., Ltd, China). The tissue sections were counterstained with hematoxylin. Negative controls for specific labeling were performed in parallel by replacing the primary antibody with a normal rabbit serum. Renal cortex sections were digitally imaged and quantitatively examined using the computer-assisted image analysis software (CX41 light microscopy; Olympus).

To quantitate the amount of chymase-positive MCs and the positive expression area of SCF and TGF- $\beta_{1}$ in the tubulointerstitial compartment, 50 fields consecutively selected in the cortical areas of the kidney were examined at a magnification of $\times 100$. Fields containing glomerular and large arteries were excluded. The analysis was made by the authors without knowledge about clinical data. Values were expressed as the average number of MCs per square millimeter of interstitium. To quantitate the expression of SCF and TGF- $\beta_{1}$ in the tubulointerstitial compartment, the average percentage of positive proportional area and the whole area was calculated by image analysis.

\section{Double Immunofluorescence Staining of Tryptase and}

TGF- $\beta_{1}$ or $S C F$

PFA-fixed frozen sections from 11th week BSA rat kidneys were thawed and washed with PBS, treated with 1\% SDS in PBS for $5 \mathrm{~min}$ for antigen retrieval, washed with PBS, and blocked in $1.5 \%$ normal horse serum in PBS (blocking solution) for $30 \mathrm{~min}$. Sections were then incubated for $1 \mathrm{~h}$ with primary antibodies. For double staining for tryptase and TGF- $\beta_{1}$, a mouse anti-tryptase antibody (1:2,000; Millipore) and a rabbit anti-TGF- $\beta_{1}$ antibody (1:100; Cell Signaling) were used; for double staining for tryptase and SCF, a mouse anti-tryptase antibody (1:2,000; Millipore) and a goat anti-SCF antibody (1:200; R\&D Systems) were used. The sections were washed with PBS and for double staining for tryptase and TGF- $\beta_{1}$ they were incubated with a mixture of donkey anti-mouse IgM Cy3 conjugated (1:400; Jackson) and donkey anti-rabbit IgG 488 conjugated (1:100: Jackson). For double staining for tryptase and SCF, sections were incubated with a mixture of donkey anti-mouse IgM Cy3 conjugated (1:400; Jackson) and donkey anti-goat IgG 488 conjugated (1:100; Jackson) in PBS for
30 min. At the final step, sections were washed with PBS and mounted with Vector Shield mounting reagent (Vector) containing $12.5 \mathrm{~g} / \mathrm{ml}$ DAPI. As a negative control, the same concentration of rabbit IgG $(4 \mathrm{~g} / \mathrm{ml})$ was used for the primary.

\section{Statistical Methods}

All statistical analyses were performed using SPSS software, version 11.0 (SPSS, Chicago, Ill., USA). Data were expressed as mean \pm SD values. $p<0.05$ was considered to be statistically significant. All variables with a normal distribution in this study were compared using ANOVA or Student's test. Significance of differences between proportions was determined by $\chi^{2}$ test. Correlations were assessed by multiple linear regressions.

\section{Results}

\section{Clinical Parameters}

24-Hour urinary protein was respectively $2.9 \pm 0.9$ and $3.1 \pm 0.9 \mathrm{mg}$ in the control group and the BSA group before the experiment started. The differences had no statistical significance ( $p>0.05)$. Compared with the saline group, rats of the BSA group demonstrated severe proteinuria which was induced in the rats by BSA injection. The level of proteinuria peaked during the 7 th week $(199.1 \pm 98.4 \mathrm{mg} / 24 \mathrm{~h})$ following the BSA injection. Then it gradually decreased until the 11 th week $(133.7 \pm 67.8$ $\mathrm{mg} / 24 \mathrm{~h}$ ). When compared with the control group, these differences were statistically significant $(\mathrm{p}<0.01)$. Furthermore, serum albumin in the BSA group was decreased compared to saline-injected controls at all time points and gradually decreased in time. Also, there was no significant difference of serum creatinine levels between the BSA group and the saline-injected controls (table 1). 

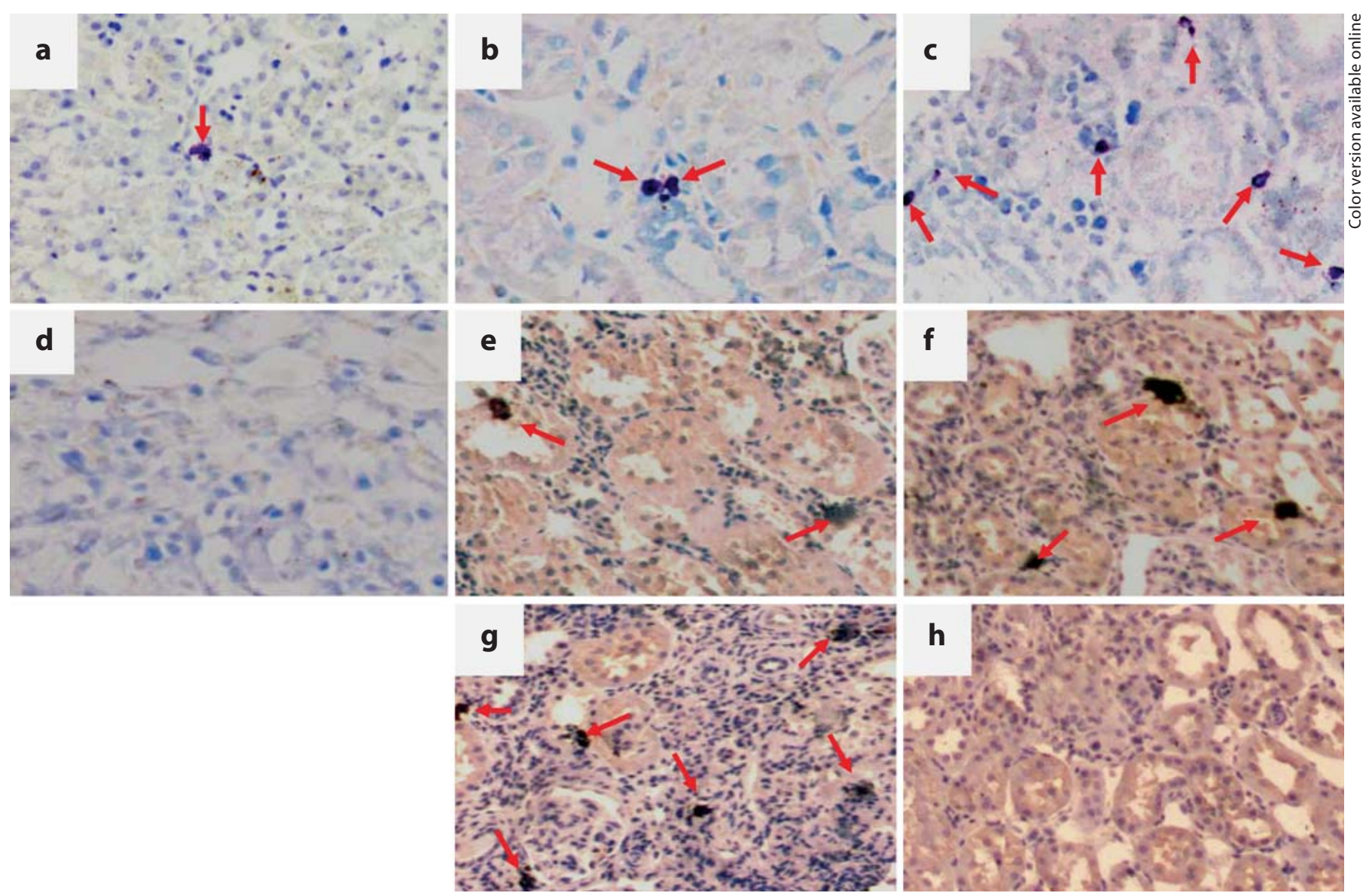

Fig. 1. Infiltration of MCs in the kidney of BSA-injected rats and saline-injected controls (a-d: toluidine blue, $\times 200$; $\mathbf{e}-\mathbf{h}$ : chymase immunohistochemistry staining, $\times 200$ ). a, e Few MCs were found in renal interstitium in the BSA-injected rats at the 3rd week. b, $\mathbf{f}$ The number of MCs obviously increased in the BSA- injected rats at the 7th week. c, $\mathbf{g}$ There were many MCs in the region of interstitial fibrosis in the BSA-injected rats at the 11th week, even migrating into the tubule. $\mathbf{d}, \mathbf{h}$ MCs cannot been observed in the renal interstitium in the saline-injected controls at the 11th week.

\section{Tubulointerstitial Injury of Renal Tissues}

Just like our previous study [12], animals receiving BSA developed a marked tubular atrophy, interstitial infiltration of inflammatory cells, and extensive fibrosis. We found that the tubulointerstitial injury was more severe in rats that received the BSA intraperitoneal injection for 11 weeks than in those that received it for 7 weeks. No significant tubulointerstitial injury was found in the rats of the saline group.

\section{Infiltration}

By using TB staining, only occasional MCs were identified in the kidneys of the rats in the saline group at all time points. In contrast, increased numbers of TB-positive MCs were noted in the tubulointerstitium of rats that had been injected with BSA at all time points, and the number of MCs gradually increased with the time of BSA injection (table 2; fig. 1). Also, MCs were localized in areas of tubular dilatation and interstitial fibrosis, but were not found in glomeruli. Using chymase staining, it was also demonstrated that the maximal number of MCs was at the 11th week in the BSA group (table 2; fig. 1). There was no difference between the number of TB-positive MCs and that of the chymase-positive MCs.

\section{Expression of Cytokine}

For SCF, the control group demonstrated almost no expression. In contrast, the BSA group showed that in the 3rd week, SCF could be detected in renal tubular epithelial cells and renal interstitium, and it increased slightly by the 7th week. At the 11th week, it obviously increased. There is a significant difference between the BSA group 

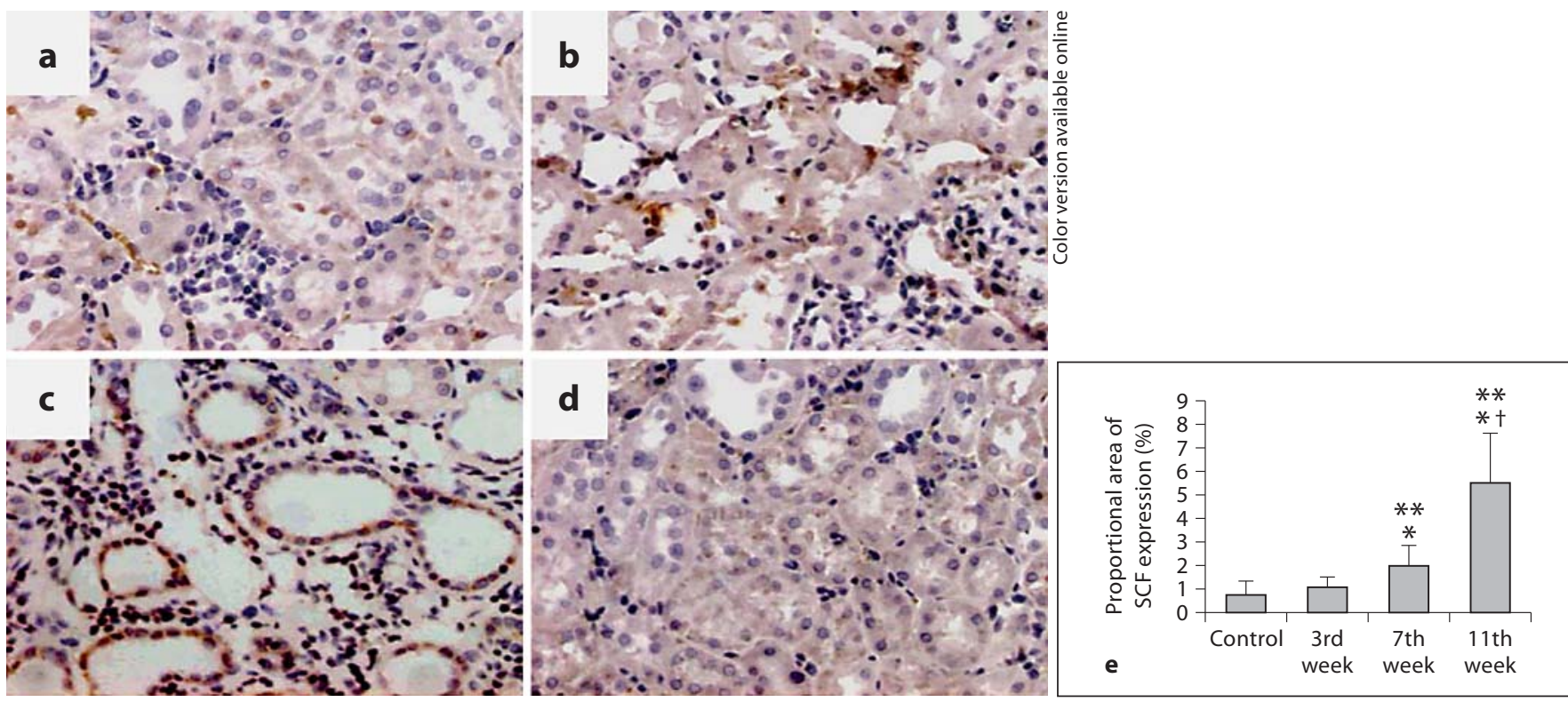

Fig. 2. Expression of SCF in the kidney of BSA-injected rats and saline-injected controls (immunohistochemistry staining, $\times 200$ ). a SCF expressed in renal tubular epithelial cells and renal interstitium in the BSA-injected rats at the 3rd week. b Expression of SCF was increased in the rats at the 7th week after BSA injection. c Expression of SCF was to a greater extent in the rats at the

11th week after BSA injection. d SCF demonstrated a very low level of expression in the tubular epithelial cells in the saline-injected controls at the 11 th week. $\mathbf{e}^{* *} \mathrm{p}<0.01$ vs. saline control; ${ }^{*} \mathrm{p}<0.05$ vs. BSA-injected rats at 3 weeks; ${ }^{\dagger} \mathrm{p}<0.05$ vs. BSA-injected rats at 7 weeks.

Table 2. MC number in BSA-injected rats and saline-injected controls (cells $/ \mathrm{mm}^{2}$, mean $\pm \mathrm{SD}$ )

\begin{tabular}{|c|c|c|c|c|c|c|}
\hline & \multicolumn{2}{|l|}{3 weeks } & \multicolumn{2}{|l|}{7 weeks } & \multicolumn{2}{|l|}{11 weeks } \\
\hline & control & BSA group & control & BSA group & control & BSA group \\
\hline TB-positive MCs & $1.10 \pm 0.99$ & $2.40 \pm 1.18^{\mathrm{a}}$ & $1.30 \pm 0.95$ & $4.62 \pm 2.72^{\mathrm{a}, \mathrm{b}}$ & $1.40 \pm 1.26$ & $9.25 \pm 3.99^{a, c}$ \\
\hline Chymase-positive MCs & $1.10 \pm 0.74$ & $2.5 \pm 1.77^{\mathrm{a}}$ & $1.20 \pm 1.03$ & $6.62 \pm 3.81^{\mathrm{a}, \mathrm{b}}$ & $1.40 \pm 0.84$ & $11.12 \pm 4.49^{\mathrm{a}, \mathrm{c}}$ \\
\hline
\end{tabular}

${ }^{\mathrm{a}} \mathrm{p}<0.05$ vs. saline-injected controls; ${ }^{\mathrm{b}} \mathrm{p}<0.05 \mathrm{vs}$. BSA-injected rats at the $3 \mathrm{rd}$ week; ${ }^{\mathrm{c}} \mathrm{p}<0.05 \mathrm{vs}$. BSA-injected rats at the 7 th week.

Table 3. Correlation between infiltration of chymase-positive MCs and interstitial fibrosis, the expression of SCF and TGF- $\beta_{1}$

\begin{tabular}{llc}
\hline & \multicolumn{2}{l}{ Chymase-positive MCs } \\
\cline { 2 - 3 } & $\mathrm{r}$ & $\mathrm{p}$ \\
\hline Interstitial fibrosis & 0.793 & 0.0001 \\
SCF & 0.868 & 0.0001 \\
TGF- $\beta_{1}$ & 0.596 & 0.0001 \\
\hline
\end{tabular}

$\mathrm{r}=$ Coefficient of correlation; $\mathrm{p}=$ level of significance. at the 7th and 11th week and the control group, respectively $(\mathrm{p}<0.05)$ (fig. 2).

For TGF- $\beta_{1}$, the control group demonstrated a very low level of expression in the tubular epithelial cells. In contrast, TGF- $\beta_{1}$ in the BSA group demonstrated higher levels of expression in renal tubular epithelial cells and renal interstitium. The difference between the BSA group and the control group is statistically significant $(\mathrm{p}<0.05)$ (fig. 3). Expression of TGF- $\beta_{1}$ increased at the 3rd week and reached a greater extent at the 11th week following BSA injection. 


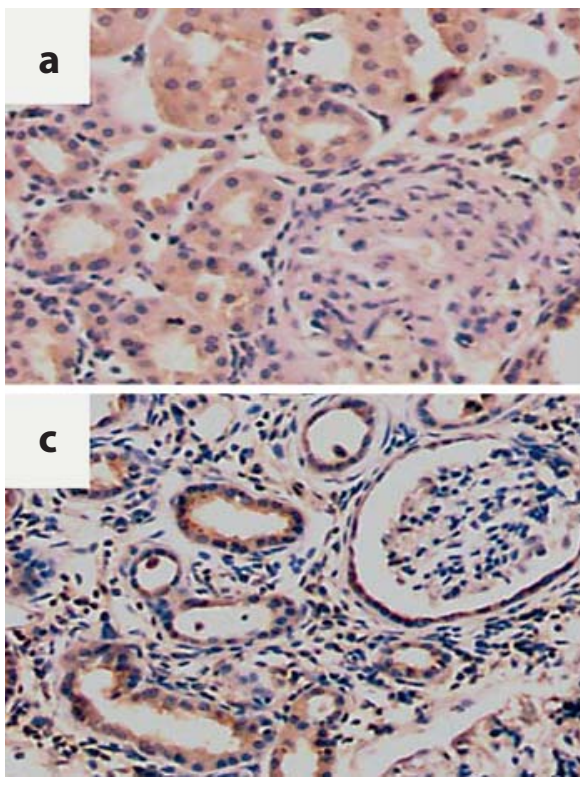

Fig. 3. Expression of TGF- $\beta_{1}$ in the kidney of BSA-injected rats and saline-injected controls (immunohistochemistry staining, $\times 200)$. a TGF- $\beta_{1}$ expressed in renal tubular epithelial cells, renal interstitium in the BSA-injected rats at the 3rd week. b Expression of TGF- $\beta_{1}$ was increased in the rats at the 7 th week after BSA in-
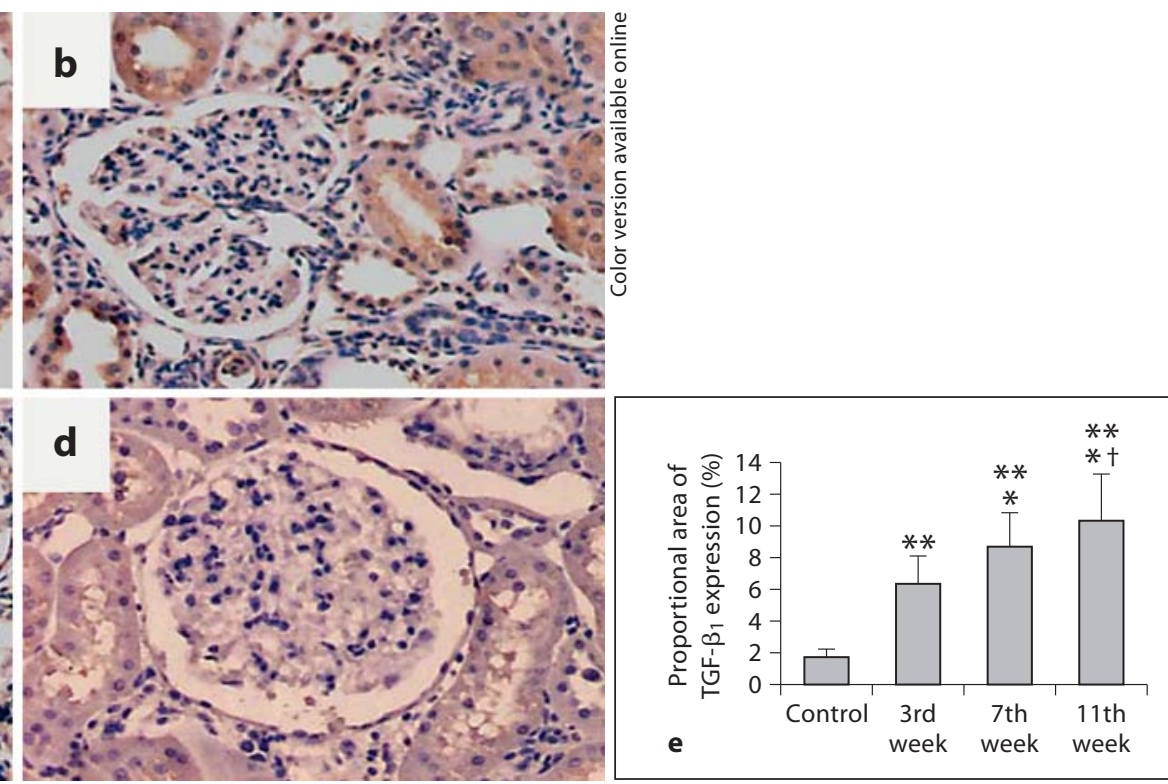

jection. $\mathbf{c}$ Expression of TGF- $\beta_{1}$ was to a greater extent in the rats at the 11th week after BSA injection. $\mathbf{d}$ TGF- $\beta_{1}$ expressed hardly in the tubular epithelial cells in the control rats at the 11th week. $\mathbf{e}^{* *} \mathrm{p}<0.01$ vs. saline-injected controls; ${ }^{*} \mathrm{p}<0.05$ vs. BSA-injected rats at 3 weeks; ${ }^{\dagger} \mathrm{p}<0.05$ vs. BSA-injected rats at 7 weeks.

decline in renal function and poor prognosis rather than the degree of glomerular damage $[1,2,13,14]$. Whereas the underlying mechanism of tubulointerstitial fibrosis has not been fully elucidated, there is wide recognition that the accumulation of infiltrating cells in the cortical interstitial space plays a pivotal role in the formation of tubulointerstitial fibrosis in progressive renal diseases. The interstitial leukocyte infiltration was proven to contribute to the renal damage by releasing inflammatory and profibrotic factors, stimulating fibroblast proliferation and matrix synthesis. However, for a long time, it was thought that the cells infiltrating the interstitium were identified as predominantly $\mathrm{T}$ lymphocytes, B lymphocytes and monocytes/macrophages $[15,16]$. MCs as a kind of inflammatory cells have been ignored or forgotten by nephrologists for a long time because they are not easily detected by routine histochemical staining. The associations between $\mathrm{MC}$ infiltration and the degree of tubulointerstitial damage in some renal diseases such as diabetic nephropathy, hypertensive nephropathy, IgA nephropathy and crescentic glomerulonephritis, support the idea that MCs are likely to be key players in the pathogenesis of interstitial fibrosis [17-20]. To our knowledge, there are no reports in the current literature that have de- 

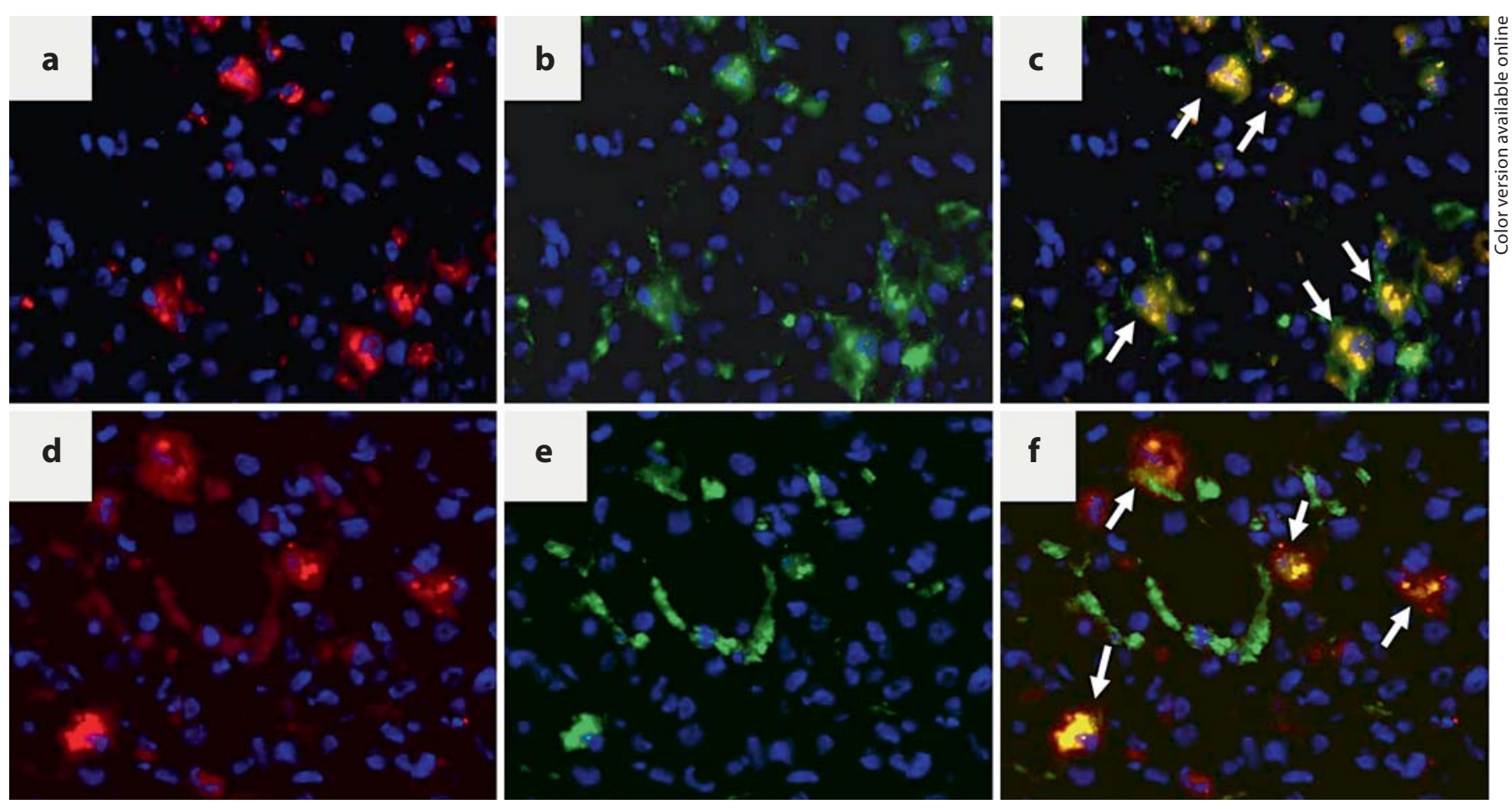

Fig. 4. Double immunofluorescence staining of tryptase and TGF- $\beta_{1}$ or SCF in rat kidneys with protein-overload nephropathy at the 11th week (DAPI: blue; all magnifications: $\times 60$ ). a Tryptase. $\mathbf{b}$ TGF- $\beta_{1}$. c Merged picture of $\mathbf{a}$ and $\mathbf{b}$. There was TGF- $\beta_{1}$ expression in some tryptase-positive MCs (arrows). d Tryptase. e SCF. $\mathbf{f}$ Merged picture of $\mathbf{d}$ and $\mathbf{e}$. There was SCF expression in some tryptase-positive MCs (arrows). scribed whether MCs participate in renal interstitial fibrosis induced by proteinuria.

Proteinuria is an important characteristic in various renal diseases and a major risk factor for the development of tubulointerstitial damage [21]. However, the pathogenesis of progressive renal tubulointerstitial damage induced by persistent proteinuria remains incompletely understood. Protein-overload nephropathy is considered an appropriate experimental model to investigate the relationship between proteinuria and interstitial damage. In our previous study [12], we had successfully constructed the model of protein-overload nephropathy. Our study showed that the proteinuria peaked during the 7 th week after the BSA injection and gradually decreased until the 11th week, but the development of renal fibrosis did not slow down. So it suggested that proteinuria itself not only directly led to renal interstitial fibrosis, but its succeeding reaction following proteinuria also played an important role in renal fibrosis.

In the present study, we investigated the distribution of MCs with TB histochemistry and chymase and tryptase staining. Staining of sections with TB is a general method to identify MCs and shows the presence of MCs. However, mature MCs contain large amounts of serine proteases such as chymase and tryptase. Positive chymase or tryptase staining therefore reflects the activation and maturation of MCs. We found that TB and chymase-positive MCs infiltrated predominantly in the interstitial and perivascular areas of the kidney with the protein-overload nephropathy, but not in the glomeruli. This finding is similar to previous reports [17-19]. Chymase-positive MCs were in line with TB-staining-positive MCs, and both of them were correlated with the severity of interstitial fibrosis. In addition, the number of MCs infiltrating the renal interstitium gradually increased with time and the quantity of proteinuria was not proportional to the number of MCs in the renal interstitium (data not shown). Just like our results, Silva et al. [22] found no correlation between MCs and proteinuria at the time of biopsy and at the end of follow-up in patients with IgA nephropathy.

MCs originate in the bone marrow, circulate in small numbers in the blood and mature in the periphery tissue 
[23]. However, how MCs are recruited and retained within the renal interstitium is still poorly understood. SCF, also known as c-kit ligand, MC growth factor or steel factor, is a key chemoattractant for MC precursor immigration and a key survival and differentiation factor for these cells. It has been reported that epithelial cells and fibroblasts can produce the soluble form of SCF which can bind to c-kit, a receptor of SCF on the membrane of the MCs $[24,25]$. In our present study, there was a high level of expression of SCF in the diseased kidneys. It appeared to localize in areas with interstitial expansion and fibrosis and was detectable predominantly in tubular cells and in interstitial infiltrating cells, while it was barely detectable in normal kidneys, and with the double immunofluorescence staining, SCF protein was also detected in some tryptase-positive MCs of the kidneys with protein-overload injury. These results are consistent with findings in human biopsies with various chronic glomerulonephritis [26]. Moreover, we found that the expression of SCF was demonstrated in the kidneys of the BSA rats at the early stage (3rd week), and the expression then gradually increased until the 11th week, which was positively correlated with the infiltration of MCs and renal interstitial fibrosis. These findings suggested that SCF might play a role in the perpetuation of $\mathrm{MC}$-mediated renal injury rather than in the initiation of the pathologic process. However, we cannot determine why SCF actually increases in the kidney tissues in the beginning, although we hypothesize that proteinuria triggers the expression and secretion of SCF in tubular epithelial cells as a starting point of tubulointerstitial fibrosis.

In addition, our study demonstrated that the expression of TGF- $\beta_{1}$ increased in the renal interstitium of the BAS-injected group. Moreover, TGF- $\beta_{1}$ expression was found in some tryptase-positive MCs and had a positive correlation with the number of MC infiltrations. In 1994 it was reported that TGF- $\beta_{1}$ serves as an extremely potent chemotactic factor that would result in the accumulation of MCs in the ongoing processes of fibrosis and angiogenesis [11]. Moreover, MCs were stimulated to release chymase, which could also activate the expression of TGF- $\beta_{1}$. It is well known that TGF- $\beta_{1}$ contributes to the development of tubulointerstitial fibrosis as an important factor $[14,27]$, so it is promising to inhibit the expression of TGF- $\beta_{1}$ by targeted blocking infiltration and activation of MCs.

In conclusion, we showed that $\mathrm{MC}$ infiltration along with the expression of both SCF and TGF- $\beta_{1}$ are closely associated with renal interstitial fibrosis in rat kidney with protein-overload nephropathy. We inferred that proteinuria might trigger MC migration to the kidney by SCF, which in turn will release chymase and tryptase, leading to the increase of TGF- $\beta_{1}$ expression, which may be involved in the initiation and progression of interstitial fibrosis. However, confirmation of such a hypothesis and the precise mechanisms involved remains to be determined by further studies. Reducing proteinuria cannot completely block the progression of renal interstitial fibrosis. Therefore, it is important to interfere with the process of renal fibrosis induced by the following effect of proteinuria. This study provides a good animal model for further exploration of the specific role of MCs in proteinuria-associated renal interstitial fibrosis. Furthermore, we should study whether blocking the recruitment and activation of MCs is a therapeutic strategy for treating interstitial renal fibrosis induced by proteinuria.

\section{Acknowledgements}

This study was supported by a Specialized Research Fund for the Doctoral Program of Higher Education of China (grant No. 20070533062) and a project by the Scientific Project of Research Center of Metabolic Syndrome in Central South University of China (grant No. DY-2008-02-03).

\section{References}

$\checkmark 1$ Hewitson TD: Renal tubulointerstitial fibrosis: common but never simple. Am J Physiol Renal Physiol 2009;296:F1239-F1244.

-2 Rodriguez-Iturbe B, Johnson RJ, HerreraAcosta J: Tubulointerstitial damage and progression of renal failure. Kidney Int Suppl 2005;99:S82-S86

3 Liu FY, Li Y, Peng YM, et al: Relationship between clinical predictors and tubulointerstitial damage in adult-onset primary nephrotic syndrome. Arch Med Res 2006;37:981-986.
4 Strutz FM: EMT and proteinuria as progression factors. Kidney Int 2009;75:475-481.

5 Nonaka Takahashi S, Fujita T, Takahashi T, et al: TGF- $\beta_{1}$ and CTGF mRNAs are correlated with urinary protein level in IgA nephropathy. J Nephrol 2008;21:53-63.

-6 Blank U, Essig M, Scandiuzzi L, et al: Mast cells and inflammatory kidney disease. Immunol Rev 2007;217:79-95.
7 Da Silva CA, Reber L, Frossard N: Stem cell factor expression, mast cells and inflammation in asthma. Fundam Clin Pharmacol 2006;20:21-39.

8 Holdsworth SR, Summers SA: Role of mast cells in progressive renal diseases. J Am Soc Nephrol 2008;19:2254-2261.

9 Li Y, Liu FY, Peng YM, et al: Mast cell, a promising therapeutic target in tubulointerstitial fibrosis. Med Hypotheses 2007;69:99103. 
10 Nilsson G, Butterfield JH, Nilsson K, Siegbahn A: Stem cell factor is a chemotactic factor for human MCs. J Immunol 1994;153: 3717-3723.

-11 Gruber BL, Marchese MJ, Kew RR: Transforming growth factor- $\beta_{1}$ mediates mast cell chemotaxis. J Immunol 1994; 152:58605867.

12 Liu FY, Li Y, Peng YM, et al: Norcantharidin ameliorates proteinuria, associated tubulointerstitial inflammation and fibrosis in protein overload nephropathy. Am J Nephrol 2008;28:465-477.

13 D’Amico G, Ferrario F, Rastaldi MP: Tubulointerstitial damage in glomerular diseases: its role in the progression of renal damage. Am J Kidney Dis 1995;26:124-132.

14 Hwang M, Kim HJ, Noh HJ, et al: TGF- $\beta_{1}$ siRNA suppresses the tubulointerstitial fibrosis in the kidney of ureteral obstruction. Exp Mol Pathol 2006;81:48-54.

15 Ninichuk V, Khandoga AG, Segerer S, et al: The role of interstitial macrophages in nephropathy of type 2 diabetic $\mathrm{db} / \mathrm{db}$ mice. Am J Pathol 2007;170:1267-1276.
16 Ko GJ, Boo CS, Jo SK, et al: Macrophages contribute to the development of renal fibrosis following ischaemia/reperfusion-induced acute kidney injury. Nephrol Dial Transplant 2008;23:842-852.

17 Okoń K, Stachura J: Increased mast cell density in renal interstitium is correlated with relative interstitial volume, serum creatinine and urea especially in diabetic nephropathy but also in primary glomerulonephritis. Pol J Pathol 2007;58:193-197.

18 Welker P, Krämer S, Groneberg DA, et al: Increased mast cell number in human hypertensive nephropathy. Am J Physiol Renal Physiol 2008;295:F1103-F1109.

19 Sakamoto-Ihara T, Suzuki Y, Kurusu A, et al Possible involvement of mast cells in renal fibrosis in patients with $\operatorname{IgA}$ nephropathy. Inflamm Res 2007;56:421-427.

20 Togawa H, Nakanishi K, Shima Y, et al: In creased chymase-positive mast cells in children with crescentic glomerulonephritis. Pediatr Nephrol 2009;24:1071-1075.

21 Zandi-Nejad K, Eddy AA, Glassock RJ, et al Why is proteinuria an ominous biomarker of progressive kidney disease? Kidney Int Suppl 2004;92:S76-S89.
22 Silva GE, Costa RS, Ravinal RC, et al: Mast cells, TGF- $\beta_{1}$ and $\alpha$-SMA expression in IgA nephropathy. Dis Markers 2008;24:181-190.

23 Okayama Y, Kawakami T: Development, migration, and survival of mast cells. Immunol Res 2006;34:97-115.

24 Huang B, Lei Z, Zhang GM, et al: SCF-mediated mast cell infiltration and activation exacerbate the inflammation and immunosuppression in tumor microenvironment. Blood 2008;112:1269-1279.

25 El Kossi MM, Haylor JL, Johnson TS, et al: Stem cell factor in a rat model of serum nephrotoxic nephritis. Nephron Exp Nephrol 2008;108:e1-e10.

26 Jones SE, Kelly DJ, Cox AJ, et al: Mast cell infiltration and chemokine expression in progressive renal disease. Kidney Int 2003; 64:906-913.

27 Murphy M, Docherty NG, Griffin B, et al: IHG- 1 amplifies TGF- $\beta_{1}$ signaling and is increased in renal fibrosis. J Am Soc Nephrol 2008;19:1672-1680. 